\title{
Candidate Mycobacterium tuberculosis genes targeted by human microRNAs
}

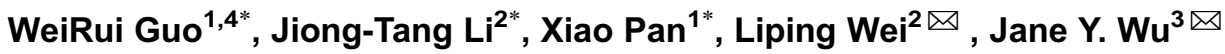 \\ 1 Institute of Biophysics, Chinese Academy of Sciences, 15 Datun Road, Beijing 100101, China \\ 2 Center for Bioinformatics, National Laboratory of Protein Engineering and Plant Genetic Engineering, College of Life Sciences, \\ Peking University, Beijing 100871, China \\ 3 Department of Neurology, Center for Genetic Medicine, Lurie Comprehensive Cancer Center, Northwestern University \\ Feinberg School of Medicine, 303 E. Superior, Chicago, IL 60611, USA \\ ${ }^{4}$ Graduate University of Chinese Academy of Sciences, 19A Yuquan Road, Beijing 100039,China \\ $\bowtie$ Correspondence: jane-wu@northwestern.edu (J. Y. Wu), weilp@mail.cbi.pku.edu.cn (L. Wei)
}

Tuberculosis (TB) remains a major health issue, causing approximately three million deaths every year (Pelicic et al., 1998). Identified by Robert Koch in 1882, Mycobacterium tuberculosis ( $M$. tuberculosis), the causative agent for tuberculosis, remains one of the most enigmatic bacteria. The interactions between $M$. tuberculosis and its environment have been extensively studied. However, our knowledge about potential $M$. tuberculosis-host interaction at the RNA level is still very limited. Here we present our preliminary finding that suggests possible interactions of human miRNAs with $M$. tuberculosis transcripts and speculate possible roles of human miRNAs in regulating macrophage-M. tuberculosis interactions. Using an miRNA target prediction software miRanda followed by analyses of cross-species conservation and miRNA expression profile, we predicted 26 candidate $M$. tuberculosis genes that may be targeted by human miRNAs expressed in the lung or macrophages, including genes related to $M$. tuberculosis drug resistance, virulence and growth. If experimentally proven, this finding could help reveal previously unsuspected host-pathogen interactions and provide a potential new direction in developing novel antituberculosis therapies.

Great progress has been made in the molecular characterization of M. tuberculosis (Smith, 2003). However, we understand very little about the molecular basis of bacterium-host interaction and molecular cellular mechanisms underlying the pathogenesis and its drug resistance. Central to the pathogenic virulence of $M$. tuberculosis is its ability to persist and multiply within alveolar macrophages after being phagocytosed, forming the primary lesion or tubercle (Houben et al., 2006). Understanding the host-pathogen interactions in tuberculosis is critical for developing new strategies to combat mycobacterial diseases.

The role of miRNAs in regulating gene expression has been increasingly appreciated in a wide spectrum of species including plants, animals and viruses. miRNAs extensively mediate antiviral defenses in plants and animals (Vance and Vaucheret, 2001; Watanabe et al., 2007). For instance, human miRNA Hsa-miR-32 restricts the replication of retrovirus PFV-1 in human cells (Lecellier et al., 2005). Viruses also use miRNAs to regulate host cellular environments. For instance, miRNA miR-K12-11 encoded by Kaposi's-sarcomaassociated herpes virus (KSHV) down-regulates an extensive set of common host mRNAs (Gottwein et al., 2007). As an intracellular bacteria, $M$. tuberculosis depends on the tolerance of host immune system for its survival and replication, which makes it susceptible to the host generegulatory mechanisms. Silencing via host miRNA might be a mechanism human macrophage employs to defend against intracellular pathogens such as $M$. tuberculosis. However, the potential roles of microRNAs in regulating bacterial genes are yet to be explored.

Different from viruses, Gram-positive mycobacteria contain an additional layer of cell wall made of peptidoglycan rich in mycolic acids, glycolipids and polysaccharides (Kolattukudy et al., 1997), and thus, poses an unusual barrier for permeability. Nonetheless, the physical integrity of the bacterial wall might have vulnerability. First, many intracellular pathogens undergo genetic regulatory responses after uptake by host cells (Finlay and Falkow, 1997). Intracellular $M$. tuberculosis expresses surface components that are not present on extracellularly grown bacilli, and these ligands

"These authors contributed equally to the work 
may facilitate bacterial entry to the host cell (McDonough et al., 1993). During the cell entry process, there might be sites of compromised physical integrity of $M$. tuberculosis cell wall. Second, macrophages inside various stresses might facilitate the entry of human miRNP into M. tuberculosis compartment. The fatty acid-rich cell envelope of $M$. tuberculosis could be damaged by exposure to alveolar surfactant (Manganelli et al., 2004), toxic peptides and proteins such as granulysin released by activated macrophages and NK cells (Dieli et al., 2001) as well as toxic free fatty acids secreted from macrophages both inside the mycobacterial phagosome and in the external environment (Akaki et al., 2000). Third, replication of $M$. tuberculosis within macrophages involves disintegration and re-establishment of its cell wall. All these may provide entry opportunities for macrophage miRNAs. Furthermore, in Arabidopsis thaliana, 21-nt siRNA, as the component of the cell-to-cell silencing signal, moves from companion cell into neighboring parenchyma cells through cell membrane and cell wall (Dunoyer et al., 2007). Moreover, studies have reported that RNAs could naturally exist in extracellular environment such as in blood (Fischer et al., 2007).

To explore possible interactions between human miRNAs and $M$. tuberculosis mRNAs, we searched the $M$. tuberculosis genome for potential target sites of known human miRNAs using miRNA target prediction method miRanda (John et al., 2004) (see details in Supplemental Fig. 1 and Data source and Methods in the Supplemental Materials). miRanda predicted 608 potential miRNA binding sites, some of which showed clustered distribution along the $M$. tuberculosis genome. We next compared three evolutionarily close genomes, M. tuberculosis H37Rv, M. tuberculosis CDC1551 and Mycobacterium leprae (M. leprae) TN and selected for further analysis only miRNA-target pairs that are conserved across three genomes. If an $M$. tuberculosis $\mathrm{H} 37 \mathrm{Rv}$ gene and its homologs in the other two genomes are targeted by the same human miRNA, we consider the interaction between the human miRNA and putative $M$. tuberculosis H37Rv target gene as "conserved". We then examined the expression profiles of human miRNAs and selected only potential targets corresponding to miRNAs expressed in human lung or macrophages (Landgraf et al., 2007). After the above two filters, our search revealed 26 candidate genes in $M$. tuberculosis that might be targets for human miRNAs (Supplemental Table 1). Of particular interest, the list included genes involved in drug-resistance and survival such as $r p s L$, $h s p R$, grpE, dnaJ1 and sodC, whose miRNA-target gene pairs and their maximum free energy are shown in Supplemental Table 2. Many candidate target genes have multiple predicted miRNA binding sites.

miRNAs' post-transcriptional regulation in eukaryotes involves multiple proteins. Although we did not find any homologs in $M$. tuberculosis of the eukaryotic Argonaute proteins containing PIWI/PAZ domains, there are other possible prokaryotic functional counterparts to the eukaryotic miRNA systems that might help human miRNAs regulate $M$. tuberculosis genes. A well-characterized prokaryotic small RNA functional pathway employs the RNA-binding protein Hfq, a conserved homohexameric ring protein closely related to $\mathrm{Sm}$ and $\mathrm{Sm}$-like proteins involved in RNA process in eukaryotes, for small RNA presentation, and RNAse E for target degradation (Sauter et al., 2003; Gottesman, 2005). In M. tuberculosis H37Rv, we found that Rv2842c contains Smlike Super-family domain (Marchler-Bauer et al., 2009) (Supplemental Fig. 2A), suggesting that it might present small RNAs; we also found that the rne gene Rv2444c could function as RNAse E for target degradation (Supplemental Fig. 2B). Recently, it was proposed that the Cas proteins in bacteria form part of the RISC-like complex to process CRISPRs (clustered, regularly interspaced short palindromic repeats) into small RNAs, analogous to the eukarytic RNAi system, in an antiphage defense system (Sorek et al., 2008). We retrieved the Cas proteins in the genome of $M$. tuberculosis from TIGR Comprehensive Microbial Resource (http://cmr.tigr.org/cgi-bin/CMR/CmrHomePage.cgi) and listed them in Supplemental Table 3.

Based on the potential interactions between human miRNAs and candidate $M$. tuberculosis target genes, we propose a novel mechanism for $M$. tuberculosis-macrophage interaction in order to elicit attention from mycobacterial research community to the regulation of small non-coding RNAs in the context of $M$. tuberculosis infection. Given the limitations of current miRNA target prediction programs, it is clear that experimental validation of predicted miRNA targets is necessary to confirm our preliminary findings. Regulation of human macrophage on $M$. tuberculosis at the RNA level remains an enigma. Anti-tuberculosis drugs select for drug resistant bacteria. Global surveillance has shown that drugresistant tuberculosis is widespread and becomes increasingly important. If host cell miRNAs are proven to play a role in regulating the intricate networks involved in human- $M$ tuberculosis interactions, it will open up a new area in both miRNA and $M$. tuberculosis research.

\section{ACKNOWLEDGMENTS}

This work was supported by the Ministry of Science and Technology program (MOST) (Grant Nos. 2009CB825402 and 2006CB911003).

\section{REFERENCES}

Akaki, T., Tomioka, H., Shimizu, T., Dekio, S., and Sato, K. (2000). Comparative roles of free fatty acids with reactive nitrogen intermediates and reactive oxygen intermediates in expression of the anti-microbial activity of macrophages against Mycobacterium tuberculosis. Clin Exp Immunol 121, 302-310.

Dieli, F., Troye-Blomberg, M., Ivanyi, J., Fournie, J.J., Krensky, A.M., Bonneville, M., Peyrat, M.A., Caccamo, N., Sireci, G., and Salerno, A. (2001). Granulysin-dependent killing of intracellular and 
extracellular Mycobacterium tuberculosis by Vgamma9/Vdelta2 $\mathrm{T}$ lymphocytes. J Infect Dis 184, 1082-1085.

Dunoyer, P., Himber, C., Ruiz-Ferrer, V., Alioua, A., and Voinnet, O. (2007). Intra- and intercellular RNA interference in Arabidopsis thaliana requires components of the microRNA and heterochromatic silencing pathways. Nat Genet 39, 848-856.

Finlay, B.B., and Falkow, S. (1997). Common themes in microbial pathogenicity revisited. Microbiol Mol Biol Rev 61, 136-169.

Fischer, S., Gerriets, T., Wessels, C., Walberer, M., Kostin, S., Stolz, E., Zheleva, K., Hocke, A., Hippenstiel, S., and Preissner, K.T. (2007). Extracellular RNA mediates endothelial-cell permeability via vascular endothelial growth factor. Blood 110 , 2457-2465.

Gottesman, S. (2005). Micros for microbes: non-coding regulatory RNAs in bacteria. Trends Genet 21, 399- 404.

Gottwein, E., Mukherjee, N., Sachse, C., Frenzel, C., Majoros, W.H., Chi, J.T., Braich, R., Manoharan, M., Soutschek, J., Ohler, U., et al. (2007). A viral microRNA functions as an orthologue of cellular miR-155. Nature 450, 1096-1099.

Houben, E.N., Nguyen, L., and Pieters, J. (2006). Interaction of pathogenic mycobacteria with the host immune system. Curr Opin Microbiol 9, 76-85.

John, B., Enright, A.J., Aravin, A., Tuschl, T., Sander, C., and Marks, D.S. (2004). Human MicroRNA targets. PLoS Biol 2, e363.

Kolattukudy, P.E., Fernandes, N.D., Azad, A.K., Fitzmaurice, A.M., and Sirakova, T.D. (1997). Biochemistry and molecular genetics of cell-wall lipid biosynthesis in mycobacteria. Mol Microbiol 24, 263-270.

Landgraf, P., Rusu, M., Sheridan, R., Sewer, A., lovino, N., Aravin, A., Pfeffer, S., Rice, A., Kamphorst, A.O., Landthaler, M., et al. (2007). A mammalian microRNA expression atlas based on small RNA library sequencing. Cell 129, 1401-1414.
Lecellier, C.H., Dunoyer, P., Arar, K., Lehmann-Che, J., Eyquem, S., Himber, C., Saib, A., and Voinnet, O. (2005). A cellular microRNA mediates antiviral defense in human cells. Science 308 , 557-560.

Manganelli, R., Provvedi, R., Rodrigue, S., Beaucher, J., Gaudreau, L., and Smith, I. (2004). Sigma factors and global gene regulation in Mycobacterium tuberculosis. J Bacteriol 186, 895-902.

Marchler-Bauer, A., Anderson, J.B., Chitsaz, F., Derbyshire, M.K., DeWeese-Scott, C., Fong, J.H., Geer, L.Y., Geer, R.C., Gonzales, N.R., Gwadz, M., et al. (2009). CDD: specific functional annotation with the Conserved Domain Database. Nucleic Acids Res 37, D205-210.

McDonough, K.A., Kress, Y., and Bloom, B.R. (1993). Pathogenesis of tuberculosis: interaction of Mycobacterium tuberculosis with macrophages. Infect Immun 61, 2763-2773.

Pelicic, V., Reyrat, J.M., and Gicquel, B. (1998). Genetic advances for studying Mycobacterium tuberculosis pathogenicity. Mol Microbiol 28, 413-420.

Sauter, C., Basquin, J., and Suck, D. (2003). Sm-like proteins in Eubacteria: the crystal structure of the Hfq protein from Escherichia coli. Nucleic Acids Res 31, 4091-4098.

Smith, I. (2003). Mycobacterium tuberculosis pathogenesis and molecular determinants of virulence. Clin Microbiol Rev 16, 463-496.

Sorek, R., Kunin, V., and Hugenholtz, P. (2008). CRISPR-a widespread system that provides acquired resistance against phages in bacteria and archaea. Nat Rev Microbiol 6, 181-186.

Vance, V., and Vaucheret, H. (2001). RNA silencing in plants-defense and counterdefense. Science 292, 2277-2280.

Watanabe, Y., Kishi, A., Yachie, N., Kanai, A., and Tomita, M. (2007). Computational analysis of microRNA-mediated antiviral defense in humans. FEBS Lett 581, 4603-4610. 